\title{
Blood flow oscillations as a signature of microvascular abnormalities
}

Elena V. Zharkikh, Irina A. Mizeva, Irina I. Makovik, Viktor V. Dremin, Evgeny A. Zherebtsov, et al.

Elena V. Zharkikh, Irina A. Mizeva, Irina I. Makovik, Viktor V. Dremin, Evgeny A. Zherebtsov, Elena V. Potapova, Andrey V. Dunaev, "Blood flow oscillations as a signature of microvascular abnormalities," Proc. SPIE 10685,

Biophotonics: Photonic Solutions for Better Health Care VI, 106854C (17 May 2018); doi: 10.1117/12.2307476

SPIE. Event: SPIE Photonics Europe, 2018, Strasbourg, France 


\title{
Blood flow oscillations as a signature of microvascular abnormalities
}

\author{
Elena V. Zharkikh ${ }^{\mathrm{a}}$, Irina A. Mizeva ${ }^{\mathrm{b}}$, Irina I. Makovik ${ }^{\mathrm{a}}$, Viktor V. Dremin ${ }^{\mathrm{a}}$, \\ Evgeny A. Zherebtsov ${ }^{c}$, Elena V. Potapova ${ }^{a}$, and Andrey V. Dunaev ${ }^{a}$ \\ ${ }^{a}$ Orel State University named after I.S. Turgenev, 95 Komsomolskaya St, Orel, 302026, Russia \\ bInstitute of Continuous Media Mechanics, Korolyov 1, Perm, 614013, Russia \\ ${ }^{\mathrm{c}}$ Aston Institute of Photonic Technologies, Aston University, Aston Triangle, Birmingham, B4 \\ $7 \mathrm{ET}, \mathrm{UK}$
}

\begin{abstract}
Laser Doppler flowmetry (LDF) was utilized for blood flow measurements. Wavelet analysis was used to identify spectral characteristics of the LDF signal in patients with rheumatic diseases and diabetes mellitus. Baseline measurements were applied for both pathological groups. Blood flow oscillations analyses were performed by means of the wavelet transform.

Higher baseline perfusion was observed in both pathological groups in comparison to controls. Differences in the spectral properties between the groups studied were revealed. The results obtained demonstrated that spectral properties of the LDF signal collected in basal conditions may be the signature of microvasculature functional state.
\end{abstract}

Keywords: laser Doppler flowmetry, blood microcirculation, vasomotions, wavelets, diabetes, rheumatic disease

\section{INTRODUCTION}

Microcirculation plays an important role in the transport of nutrients, oxygen, hormones and the release of metabolic products. Evaluation of the microcirculatory system disorders can reveal manifestations of developing cardiovascular diseases (CVD), preceding violations in larger vessels. ${ }^{1}$ Previous works have shown that systemic microcirculatory dysfunction can be associated with risk factors for coronary heart disease,${ }^{2}$ hypertension, ${ }^{3}$ insulin resistance. ${ }^{4}$

In recent decades there has been a steady increase in the interest of researchers to the microcirculatory disorders in patients with rheumatic diseases (RD) and diabetes mellitus (DM) due to the significant role of microcirculation in the pathogenesis of these diseases. RD lead to morphological changes in the microcirculatory bed, such as rarefaction of the capillary network, capillaries asymmetry and the appearance of megacapillaries. ${ }^{5}$ Chronic hyperglycemia and insulin resistance in DM cause increased vascular permeability, disruption of vascular tone, causing structural and functional changes in capillaries and arterioles. The earliest, usually reversible, manifestation of these diseases is the development of microcirculatory dysfunction due to endothelial damage, excessive expression of certain adhesion molecules and other factors.

The microcirculatory function can be evaluated by means of various optical non-invasive methods, among which laser Doppler flowmetry (LDF) is one of the most widespread. ${ }^{6}$ It allows temporary monitoring of the microcirculation, which indirectly characterizes the vasomotions resulting from the functioning of the blood microcirculation system. ${ }^{7}$ However, the major limitations of LDF are the heterogeneous distribution of microcirculation and its oscillatory components across skin surface and a lack of a standardized approach to combat these sources of variability in order to compare the LDF data obtained under different experimental conditions.

The signal recorded by LDF is the superposition of several oscillatory components corresponding to specific physiological mechanisms. Traditionally, blood flow fluctuations were considered to be a source of non-reproducibility,

Send correspondence to Elena Zharkikh

E-mail: ev.zharkikh@gmail.com

Telephone: +79534740686

Biophotonics: Photonic Solutions for Better Health Care VI, edited by Jürgen Popp,

Valery V. Tuchin, Francesco Saverio Pavone, Proc. of SPIE Vol. 10685, 106854C

(C) 2018 SPIE · CCC code: $0277-786 X / 18 / \$ 18 \cdot$ doi: $10.1117 / 12.2307476$

Proc. of SPIE Vol. 10685 106854C-1 
arising from stochastic processes. ${ }^{8}$ At the same time, the use wavelet spectral decomposition of long-term perfusion records allows one to reveal the oscillatory components corresponding to the specific physiological mechanisms. Previous studies have shown that these oscillatory components indicate the influence of cardiac $(0.6-1.6 \mathrm{~Hz})$, respiratory $(0.145-0.6 \mathrm{~Hz})$, myogenic $(0.052-0.145 \mathrm{~Hz})$, neurogenic $(0.021-0.052 \mathrm{~Hz})$ and endothelial $(0.0095-0.021 \mathrm{~Hz})$ activities. ${ }^{8,9}$

The aim of this work was to review our last studies on spectral properties of LDF samples to answer the question whether its form can be a signature of functional microvascular abnormalities.

\section{MATERIALS AND METHODS}

\subsection{Subjects with rheumatic diseases}

In our previous work ${ }^{10}$ we examined the possibility of detecting microcirculatory disorders in patients with RD using wavelet analysis of LDF signals. Sixty subjects with RD ( $55 \pm 14$ years, 12 men and 48 women) were involved in this study. Thirty two clinically healthy participants ( $22 \pm 2$ years, 16 men and 16 women) volunteered for the study. Data are means \pm SD unless otherwise indicated. During the study participants were sitting with arms placed on a table at the heart level. The LDF sensor was located on the volar surface of the third finger of the right hand. The study lasted $5 \mathrm{~min}$ and included registration of the LDF signal under the basal conditions. Experimental systems LAKK-OP and LAKK-M (SPE LAZMA Ltd., Russia) were used for carrying out experimental studies.

\subsection{Subjects with diabetes}

The following study involved 17 patients with type $1 \mathrm{DM}$ and 40 conditionally healthy volunteers who constituted the control group, according to the inclusion criteria. ${ }^{11}$ The demographic data for the group of patients were the following: age $35 \pm 9$ years, 10 men and 7 women. The control group included 26 men and 17 women of $39 \pm 9$ years. The LDF channel of the LAZMA-SD device was used for carrying out experimental studies.

The experiment included a study of microcirculation within 4 minutes in basal conditions. During entire protocol participants resting supine with LDF probe placed on the dorsum of the foot on the plateau between the 1st and 2nd metatarsals. The experimental foot was positioned at heart level. Figure 1 presents the principle of measurement and a typical example of LDF record obtained during the studies.
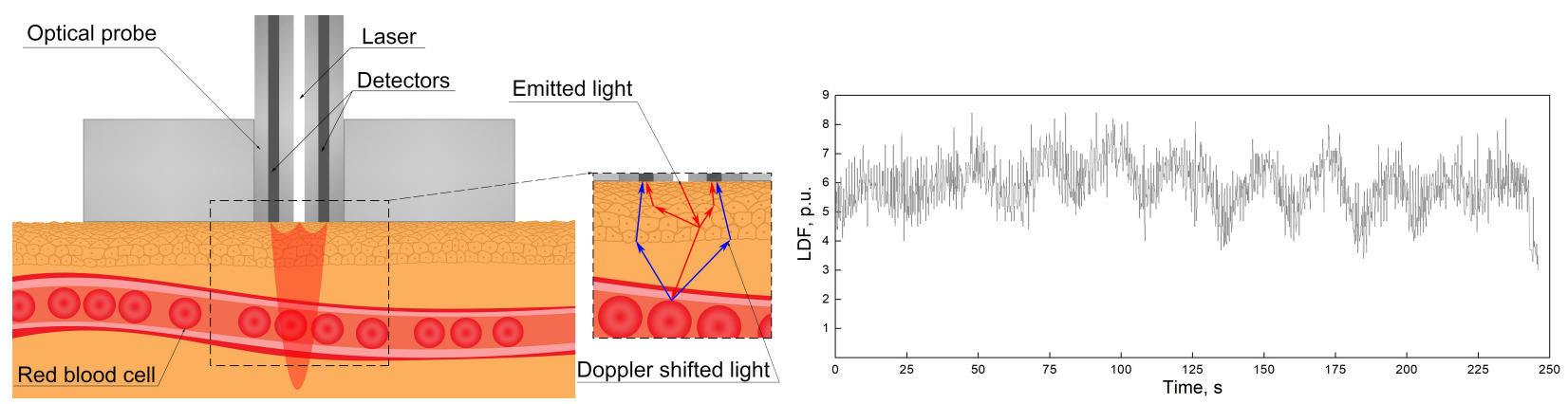

Figure 1. The principle of measurement (left panel) and a typical view of the obtained LDF signal (right panel) for a 4-minute recording

\subsection{Ethical approval}

These studies were approved by the Ethical Committee of the Orel Regional Clinical Hospital. The studies were performed in accordance with the principles outlined in the 2013 Declaration of Helsinki by the World Medical Association. Informed consent was obtained from each participant prior to the study. 


\subsection{Data analysis}

The obtained LDF signals were decomposed by means of the wavelet transform. ${ }^{10}$ The calculation of the wavelet coefficients was performed for the frequency range 0.01 to $2 \mathrm{~Hz}$ with the logarithmic partitioning on 50 frequencies. The integral wavelet spectra were averaged over each group. Energy distribution was obtained for each frequency band and compared in healthy and pathological groups.

The differences between healthy and pathological groups were examined using the Mann-Whitney test. The level of the significance was set at 0.05 . The data processing was performed by Mathematica 8.0, Wolfram research.

\section{RESULTS AND DISCUSSION}

In the presented studies, the raw wavelet spectra avoiding post-processing, were compared. Figure 2 presents the wavelet spectra averaged over groups of patients with RD and controls (left panel) and patients with DM and control group (right panel) at basal conditions. As it could be seen from the data presented, the analysis
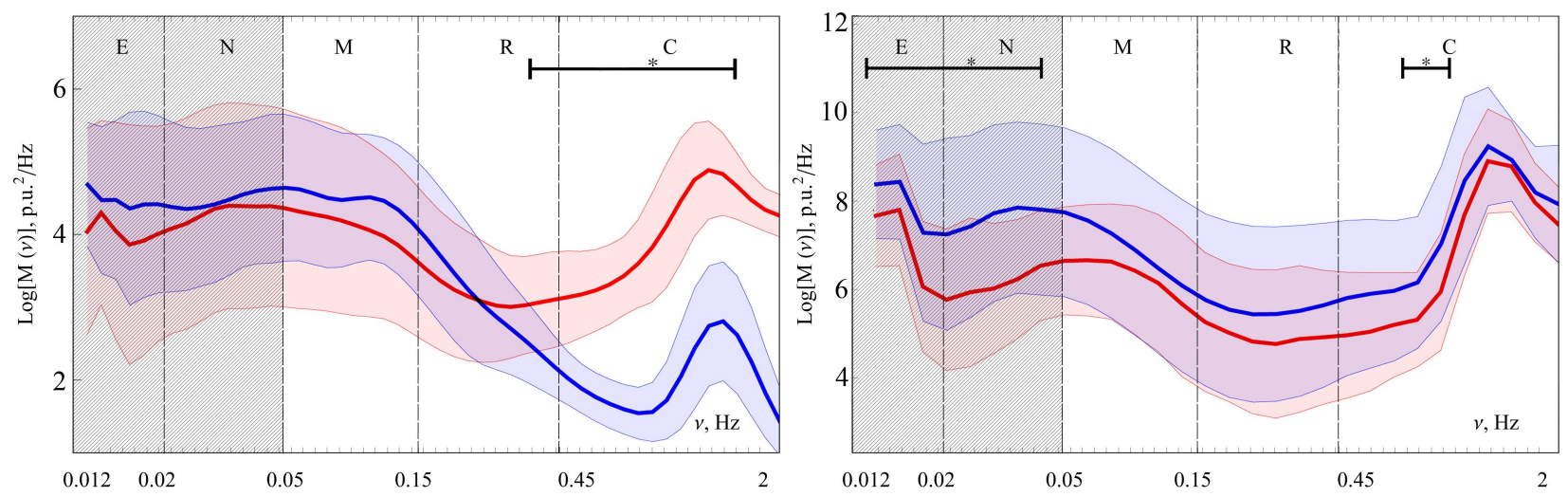

Figure 2. Averaged spectra of LDF records. Blue areas correspond to the control groups, red patients (patients with rheumatic diseases on the left panel and patients with DM on the right). The thick color line in the middle of the region corresponds to the average value, the thin lines along the edges of the region are standard deviations from the sample. Thick black lines in the upper parts of the plot indicate the frequency band, where $M(\nu)$ is significantly different $(p<0.05)$.

revealed a significant difference in the spectral characteristics of blood flow oscillations among groups of healthy volunteers and pathological groups. Patients with RD had significantly higher averaged perfusion in basal conditions in comparison to controls. Higher amplitudes of oscillations in the frequency range above $0.1 \mathrm{~Hz}$ were observed for patients with RD. The results obtained could be explained by the structural and functional changes in microcirculation occurring in the development of RD. As it is known from the previous studies, RD are characterized by an increase in the size of the capillaries, angiogenesis and the appearance of megacapillaries. ${ }^{5}$ The average level of perfusion in patients with DM was slightly higher than in the control group, but the parameters did not reach statistically significant difference. These results correspond to previous studies in this area, where blood perfusion was lower in diabetic subjects, but did not differ statistically. ${ }^{12,13}$ Subjects with DM had impaired amplitude of blood flow oscillations in the frequency band 0.012-0.045 Hz, which corresponds to endothelial and neurogenic vascular tone regulating mechanisms.

The energy of $1 \mathrm{~Hz}$ pulsations was significantly lower in patients in comparison to control group. Increased arterial stiffness and abnormal endothelial reactivity may be the cause of reduced cardiac and endothelial oscillation amplitudes respectively.

The proposed diagnostic approach can be applied not only to rheumatic diseases or diabetes but also to other pathologies. Previously, an analysis of cutaneous blood flow oscillations, recorded in basal conditions by the LDF, was used for non-invasive identification of malignant skin melanoma. ${ }^{8}$

The sufficient study limitation of this work concerns difference in the probe location. These places has different morphological structures, but as it was demonstrated in Sorelli $2017^{14}$ spectral properties in different locations 
have not so dramatic difference. Moreover we can compare controls in studies under consideration. Also we have poor statistic for low-frequency oscillations due to short records.

\section{CONCLUSIONS}

In this study laser Doppler flowmetry has been successfully applied for the assessment of variations in spectral characteristics of blood flow in the groups of patients with $\mathrm{RD}, \mathrm{DM}$ and healthy volunteers.

When carrying out baseline records of the signal in patients with $\mathrm{RD}$, increased values of the oscillations at frequencies above $0.1 \mathrm{~Hz}$ were revealed. A group of patients with diabetes showed reduced oscillation amplitudes in the low-frequency $(0.012-0.045 \mathrm{~Hz})$ range and at a frequency of about $1 \mathrm{~Hz}$.

The results obtained during the research demonstrate the ability of LDF to provide useful information for the diseases classification in a non-invasive manner even in basal conditions.

The proposed diagnostic approach and subsequent data processing have a potential to underlie a clinical method for diagnostics and monitoring of microvascular disorders of different etiologies and can be applied in screening to detect microcirculation system disorders and a qualitative define the characteristics of the disorders.

\section{ACKNOWLEDGMENTS}

This work was supported by the Russian foundation for basic research RFBR 17-41-590560. Evgeny Zherebtsov kindly acknowledges funding from the European Union's Horizon 2020 research and innovation programme under the Marie Sklodowska-Curie grant agreement No. 703145.

Special thanks are extended to doctors Khakhicheva L.S., Alimicheva E.A., Masalygina G.I., and Muradyan V.F. of the Orel Regional Clinical Hospital for providing useful advice and help.

\section{REFERENCES}

[1] Kerekes, G., Soltsz, P., Nurmohamed, M., Gonzalez-Gay, M., Turiel, M., Vgh, E., Shoenfeld, Y., McInnes, I., and Szekanecz, Z., "Validated methods for assessment of subclinical atherosclerosis in rheumatology," Nature Reviews Rheumatology 8(4), 224-234 (2012).

[2] Ijzerman, R., De Jongh, R., Beijk, M., Van Weissenbruch, M., Delemarre-van De Waal, H., Sern, E., and Stehouwer, C., "Individuals at increased coronary heart disease risk are characterized by an impaired microvascular function in skin," European Journal of Clinical Investigation 33(7), 536-542 (2003).

[3] Levy, B., Ambrosio, G., Pries, A., and Struijker-Boudier, H., "Microcirculation in hypertension: A new target for treatment?," Circulation 104(6), 735-740 (2001).

[4] Wheatcroft, S., Williams, I., Shah, A., and Kearney, M., "Pathophysiological implications of insulin resistance on vascular endothelial function," Diabetic Medicine 20(4), 255-268 (2003).

[5] De Angelis, R., Grassi, W., and Cutolo, M., "A growing need for capillaroscopy in rheumatology," Arthritis Care and Research 61(3), 405-410 (2009).

[6] Daly, S. and Leahy, M., "'go with the flow ': A review of methods and advancements in blood flow imaging," Journal of Biophotonics 6(3), 217-255 (2013).

[7] Dunaev, A., Sidorov, V., Krupatkin, A., Rafailov, I., Palmer, S., Stewart, N., Sokolovski, S., and Rafailov, E., "Investigating tissue respiration and skin microhaemocirculation under adaptive changes and the synchronization of blood flow and oxygen saturation rhythms," Physiological Measurement 35(4), 607-621 (2014).

[8] Lancaster, G., Stefanovska, A., Pesce, M., Marco Vezzoni, G., Loggini, B., Pingitore, R., Ghiara, F., Barachini, P., Cervadoro, G., Romanelli, M., and Rossi, M., "Dynamic markers based on blood perfusion fluctuations for selecting skin melanocytic lesions for biopsy," Scientific Reports 5, 12825 (2015).

[9] Stefanovska, A., Bracic, M., and Kvernmo, H. D., "Wavelet analysis of oscillations in the peripheral blood circulation measured by laser doppler technique," IEEE transactions on bio-medical engineering 46(10), 1230-1239 (1999).

[10] Mizeva, I., Makovik, I., Dunaev, A., Krupatkin, A., and Meglinski, I., "Analysis of skin blood microflow oscillations in patients with rheumatic diseases," Journal of Biomedical Optics 22(7), 070501 (2017). 
[11] Dremin, V., Zherebtsov, E., Sidorov, V., Krupatkin, A., Makovik, I., Zherebtsova, A., Zharkikh, E., Potapova, E., Dunaev, A., Doronin, A., Bykov, A., Rafailov, I., Litvinova, K., Sokolovski, S., and Rafailov, E., "Multimodal optical measurement for study of lower limb tissue viability in patients with diabetes mellitus," Journal of Biomedical Optics 22(8), 085003 (2017).

[12] Walther, G., Obert, P., Dutheil, F., Chapier, R., Lesourd, B., Naughton, G., Courteix, D., and Vinet, A., "Metabolic syndrome individuals with and without type 2 diabetes mellitus present generalized vascular dysfunction: Cross-sectional study," Arteriosclerosis, Thrombosis, and Vascular Biology 35(4), 1022-1029 (2015).

[13] Fuchs, D., Dupon, P., Schaap, L., and Draijer, R., "The association between diabetes and dermal microvascular dysfunction non-invasively assessed by laser doppler with local thermal hyperemia: A systematic review with meta-analysis," Cardiovascular Diabetology 16(1) (2017).

[14] Sorelli, M., Stoyneva, Z., Mizeva, I., and Bocchi, L., "Spatial heterogeneity in the time and frequency properties of skin perfusion," Physiological Measurement 38(5), 860-876 (2017). 